\title{
A Randomized Controlled Trial of Postoperative Thoracic Epidural Analgesia Versus Intravenous Patient Controlled Analgesia after Major Hepatopancreatobiliary Surgery
}

\author{
Thomas A. Aloia, MD ${ }^{1}$, Bradford J. Kim, MD, MHS ${ }^{1}$, Yun Shin Segraves-Chun, MD ${ }^{1}$, Juan P. \\ Cata, MD², Mark J. Truty, MD, MS ${ }^{3}$, Qiuling Shi, MD, PhD, MSc ${ }^{4}$, Alexander Holmes, MD², \\ Jose M. Soliz, MD², Keyuri U. Popat, MD², Thomas F. Rahlfs, MD², Jeffrey E. Lee, MD ${ }^{1}$, Xin \\ Shelley Wang, MD, MPH ${ }^{3}$, Jeffrey S. Morris, PhD $^{5}$, Vijaya N.R. Gottumukkala, MB, BS, MD ${ }^{3,}{ }^{*}$, \\ and Jean-Nicolas Vauthey, MD ${ }^{1, *}$ \\ ${ }^{1}$ Department of Surgical Oncology, The University of Texas MD Anderson Cancer Center, \\ Houston, Texas \\ 2Department of Anesthesiology and Perioperative Medicine, The University of Texas MD \\ Anderson Cancer Center, Houston, Texas \\ ${ }^{3}$ Department of Surgery, Mayo Clinic, Rochester, Minnesota \\ ${ }^{4}$ Department of Symptom Research, The University of Texas MD Anderson Cancer Center, \\ Houston, Texas \\ ${ }^{5}$ Department of Biostatistics, The University of Texas MD Anderson Cancer Center, Houston, \\ Texas
}

\begin{abstract}
Objectives-The primary objective of this randomized trial was to compare thoracic epidural analgesia (TEA) to intravenous patient controlled analgesia (IV-PCA) for pain control over the first 48 hours after hepatopancreatobiliary (HPB) surgery. Secondary endpoints were patientreported outcomes, total narcotic utilization and complications.
\end{abstract}

Summary of Background Data-Although adequate postoperative pain control is critical to patient and surgeon success, the optimal analgesia regimen in HPB surgery remains controversial.

Methods-Using a 2.5:1 randomization strategy, 140 patients were randomized to TEA ( $\mathrm{N}=106)$ or IV-PCA (N=34). Patient-reported pain was measured on a Likert scale (0-10) at standard time intervals. Cumulative pain area under the curve (AUC) was determined using the trapezoidal method.

\footnotetext{
Address all correspondence and reprint requests to: Thomas A. Aloia, MD, University of Texas MD Anderson Cancer Center, Department of Surgical Oncology, 1400 Herman Pressler Drive, Unit 1484, Houston, TX 77030, Phone: (713) 563-0189, Fax: (713) 745-6287, taaloia@mdanderson.org.

*Drs. Gottumukkala and Vauthey equally share senior authorship.

Financial Disclosures: None

National Meeting Presentation: These data were presented at the $2017137^{\text {th }}$ Annual American Surgical Association Annual Meeting
} 
Results-Between the study groups key demographic, comorbidity, clinical and operative variables were equivalently distributed. The median AUC of the postoperative time 0 to 48 hour pain scores was lower in the TEA group (78.6 vs 105.2 pain-hours, $\mathrm{p}=0.032$ ) with a $35 \%$ reduction in patients experiencing $\geq 7 / 10$ pain $(43 \%$ vs $66 \%, \mathrm{p}=0.05)$. Patient-reported outcomes and total opiate use further supported the benefit of TEA on patient experience. Anesthesia related events requiring change in analgesic therapy were comparable ( $12.2 \%$ vs. $2.9 \%$, respectively, $\mathrm{p}=0.187$ ). Grade $\geq 3$ surgical complications, median length of stay ( 6 days vs 6 days), readmission (1.9\% vs $3.1 \%$ ), and return to the OR ( 0.9 vs $3.1 \%$ ) were similar (all $p>0.05$ ). There were no mortalities in either group.

Conclusions-In major HPB surgery, TEA provides a superior patient experience through improved pain control and less narcotic use, without increased length of stay or complications.

\section{Keywords}

hepatectomy; pain management; pancreaticoduodenectomy; patient reported outcomes (PROs); quality of life

\section{INTRODUCTION}

As the safety of hepatopancreaticobiliary surgery has significantly improved, the practice has grown in cases and complexity.[1-14] Compared to traditional open surgical procedures, minimally invasive laparoscopic surgery has led to decreased postoperative pain.[15-17] However, in the majority of hepatic and pancreatic resections, a minimally invasive approach is not technically feasible; and postoperative pain continues to be poorly controlled despite high narcotic doses, with adverse impact on patient experience and patient outcomes.[18]

Inadequate pain control after open abdominal procedures may have both primary and secondary impacts on patient outcomes. In the initial postoperative period, inadequate pain control contributes to the neuroendocrine stress response, increased complications and prolonged length of stay.[19,20] These adverse initial outcomes can delay overall recovery, limiting the patient's ability to return to normal levels of function, and in the case of cancer patients, the ability to return to intended oncologic therapy (RIOT).[21] This is particularly relevant in hepatopancreatobiliary surgery, where procedures can be disabling and most patients undergo oncologic resection. Effective pain control is, therefore, imperative in these patients. Yet, the best modality for pain control after hepatopancreaticobiliary resection remains unclear.

Two of the most common analgesic modalities used after inpatient abdominal surgery are thoracic epidural analgesia (TEA) using local anesthetic agents and/or narcotics, and intravenous patient controlled analgesia (IV-PCA) using narcotics. Multiple non-randomized comparisons have been reported, however, relatively few level 1 data from randomized trials are available.[22-28] Currently, the advantages and disadvantages between these two strategies continue to be debated, and the most effective strategy has not been determined. The objective of this randomized control trial (RCT) was to compare the efficacy of pain control and safety between TEA and IV-PCA. 


\section{METHODS}

\section{Patients}

This study was conducted between February 2012 and September 2016 at the University of Texas MD Anderson Cancer Center. The Ethical Committee on Clinical Investigation approved this RCT and it was registered in accordance with ClinicalTrials.gov (NCT01438476). A written informed consent was obtained before surgery from all participating patients.

Eligible patients were 18 years of age or older undergoing liver or pancreatic resection for malignancy. Both minor and major hepatectomies were included in the RCT. Major hepatectomy was defined by the Brisbane 2000 terminology as resection of 3 or more Couinaud segments.[29-32]

All patients were required to have adequate coagulation function within 30 days of surgery, defined as platelet count $\geq 100,000 / \mathrm{mL}$, INR $\leq 1.5$, and partial thromboplastin time (PTT) $\leq$ 40 seconds. Patients with fever, evidence of infection, or other coexisting medical conditions that would preclude epidural placement were excluded. Other exclusions included: history of chronic pain, long-term narcotic use, history of anaphylaxis to local anesthetics or narcotics, previous or current neurologic disease affecting the hemithorax or below, bleeding diathesis or coagulopathy, major open abdominal/thoracic surgery in the previous 30 days under general anesthesia (except total intravenous anesthesia), ongoing use or planned perioperative use of anticoagulants (not including deep vein thrombosis prophylaxis). Patients with educational, psychiatric (untreated or poorly controlled schizophrenia, major depression, or bipolar disorder), or communication (language) barriers that would preclude accurate assessment of postoperative pain and/or ability to answer questions about pain were not eligible.

\section{Randomization}

After providing written informed consent, patients undergoing resection were randomly assigned on the morning of surgery to the TEA or IV-PCA group with a 2.5 TEA: 1 IV-PCA randomization strategy.

\section{Analgesic Regimens}

All patients randomized to TEA had a thoracic epidural catheter placed preoperatively in the holding area or the operating room. Epidural catheters were placed between the $5^{\text {th }}$ and $10^{\text {th }}$ thoracic interspinous levels using standard technique according to institutional practices.

After a negative test dose, a bolus of $10 \mathrm{mcg} / \mathrm{kg}$ of epidural hydromorphone (maximum of 1 $\mathrm{mg}$ ) was administered prior to securing the catheter. All epidural catheters were tested for correct placement and level of block was documented. Prior to surgical incision, 3-10 mL of $2 \%$ lidocaine was administered incrementally through the epidural catheter to establish a block. Continuous analgesia was subsequently achieved with an epidural infusion of bupivacaine $0.075 \%$ and hydromorphone $5 \mathrm{mcg} / \mathrm{mL}$ at $5-8 \mathrm{ml} / \mathrm{hr}$. Secondary alternatives to hydromorphone were fentanyl and bupivacaine. Infusion rates on the surgical floor were 5$8 \mathrm{~mL} / \mathrm{hr}$ with an added patient controlled epidural analgesia dose of $3 \mathrm{~mL}$ every 10 minutes 
as needed (not to exceed $6 \mathrm{~mL} / \mathrm{hr}$ ). Infusions and catheters were discontinued when patients were successfully tolerating a diet (or tube feeds) and could be safely transitioned to oral/ enteral analgesics.

For patients randomized to the IV-PCA group, intraoperative intravenous narcotics were titrated by the Anesthesia team until the patient was awake and alert in the post-anesthesia care unit, and then IV-PCA infusion was initiated. Hydromorphone was the opioid of choice unless the patient had allergic reaction or other contraindications. Secondary alternatives included fentanyl or morphine. The initial recommended starting dose settings included: no basal rate, $0.2 \mathrm{mg}$ every 10 minutes of demand dosing, and a $0.5 \mathrm{mg}$ nursing bolus every 1 hour as needed for additional pain control.

Adjunctive oral, rectal, or intravenous non-narcotic analgesics (ie. Acetaminophen or nonsteroidal) were utilized at the discretion of the Acute Pain Service and/or primary surgical team for both the TEA and IV-PCA groups. In cases with inadequate pain control, cross-over between arms was permitted, although all patient outcomes were assessed within randomized groups on an intention-to-treat basis.

\section{Study Endpoints}

The primary endpoint was the area under the curve (AUC) pain score during the first 48 hours after surgery. Patient-reported pain scores were assessed in both groups with a numeric/visual pain scale (0-10) at multiple time points per day that started immediately in the post-operative care unit and concluded after post-operative day (POD) 5 . With pain scores collected at specific time points, an Area Under the Curve for pain was calculated for the first 48 hours after surgery (AUC 48) using the trapezoidal method. Additionally, episodes of severe pain, defined as any pain score response of 7 or greater, were recorded. Secondary study endpoints included patient-reported functional outcomes, narcotic use over the first 5 post-operative days, surgical complications, analgesic related complications and length of stay.

To calculate total narcotic use, all medication doses were converted into oral morphine equivalents (OME) using previously reported conversions.[33] When calculating OME, narcotics dosed through the TEA were considered equivalent (1:1) to IV narcotic dosing administered either through intravenous line or IV-PCA.

Surgical and analgesic complication data were collected in real time and retrospectively graded with the Modified Accordion Severity Grading System (MAGS).[34] Each complication was categorized into its appropriate system: neurology, cardiovascular, pulmonary, gastrointestinal, renal, wound, sepsis, and other. In addition, post-operative bile leak and liver failure was specifically included for analysis using standard definitions.[35,36] Severe complications were defined as a MAGS 3-5. All complications were reviewed by two surgical oncology department staff members. Intraoperative and postoperative administration of packed red blood cells was considered a transfusion. The postoperative transfusion period included any time after the operation until hospital discharge. For patients with TEA, the Acute Pain Service monitored the epidural site daily for evidence of hematoma, cellulitis, abscess, cerebrospinal fluid leak, and malfunction. Analgesic 
complication was defined as any event that required a discontinuation of the analgesic modality being utilized.

Several validated patient-reported outcome tools were administered to further measure pain, satisfaction with pain control, quality of life and recovery of function. The Short Form (SF)-8 health survey [37-39] and SF-36 [40-42] were both administered preoperatively. Approximately 24 hours following surgery, the SF-8 was repeated, while the SF-36 was administered near day of hospital discharge. Quality of recovery and patient satisfaction were measured with the Health Outcomes Recovery Survey (HORS) [43] with daily administration on the first 5 post-operative inpatient days. In this survey, patients were asked "how satisfied or unsatisfied are you with overall performance of your pain medication?" This patient satisfaction score was measured on a scale from 1-7: 1. Very satisfied, 2. Somewhat satisfied, 3. Slightly satisfied, 4 . Neither satisfied nor unsatisfied, 5. Slightly unsatisfied, 6. Somewhat unsatisfied, 7. Very unsatisfied.

\section{Data Collection}

For all patients, data was prospectively collected in real-time. Variables collected included patient demographics, pain scores, perioperative clinical information, and complications. Patient-reported pain assessment began in the post anesthesia care unit (PACU) by the nursing staff approximately every hour until the patient was transferred to their designated inpatient care units. In the overnight recovery suite and general floor, pain was assessed by nursing staff approximately every 4 hours; while in the surgical intensive care unit (SICU), pain was assessed by the nursing staff every hour.

\section{Statistical Analysis}

The distribution of each continuous variable was summarized by its mean \pm standard deviation and/or median with range (R)/interquartile range (IQR). The distribution of each categorical variable was summarized in terms of its frequency and percentage. Continuous variables were compared between treatment groups by Wilcoxon rank sum tests, and for the categorical variables, the comparison between treatments was conducted with Fisher's Exact tests. To assess the relative impact of patient, surgical and analgesia variables on study endpoints, univariate and multivariate regression models were developed. Backward stepwise model selection was used to obtain the final regression model for the primary endpoint of AUC48. Piecewise polynomial regression was used to characterize mean pain score trends over time with $95 \%$ confidence bands representing interval estimators that characterize the extent of uncertainty at significance level 0.05 over the follow-up time domain observed in our study population. AdaptFitOS version 0.62 package was utilized in $\mathrm{R}$ version 3.1.3 to compute the confidence bands. Other statistical analyses were performed using SPSS Statistics 21 (IBM, Armonk, NY). All tests were two-sided.

\section{RESULTS}

During the study period, 178 screened patients were initially eligible for inclusion to the RCT. A Consort flow diagram of this RCT is shown in Figure 1.[44] Among the 178 eligible patients, 38 patients were excluded due to withdrawal of consent, development of exclusion 
criteria and/or the finding of unresectable disease at surgery. The study concluded with 140 patients eligible for analysis, with 106 patients allocated to the TEA group and 34 patients allocated to the IV-PCA group.

\section{Patient Characteristics}

Table 1 reports the distribution of patient characteristics, preoperative and perioperative variables between the TEA and IV-PCA groups. 136 patients had a liver resection and 4 patients had a major pancreas resection (3 pancreaticoduodenectomies and 1 total pancreatectomy). Oncologic indications for resection included colorectal liver metastases $(88,62.9 \%)$, cholangiocarcinoma $(13,9.3 \%)$, hepatocellular carcinoma $(9,6.4 \%)$, gallbladder adenocarcinoma $(7,5 \%)$, neuroendocrine tumor $(5,3.6 \%)$, and other histologies $(17,12.1 \%)$. There were no differences observed in preoperative clinical or perioperative features between TEA and IV-PCA groups.

\section{Primary Endpoint: Postoperative Pain Experience}

The median length of time on the study pain control modality was similar between randomized groups (TEA: 4 days, IQR 3-4 days vs. IV-PCA: 4 days, IQR 3-5 days, $\mathrm{p}=0.597$ ). For all patients, the overall median AUC 48 was 86.4 pain-hours (Range: 0267.3). The median AUC 48 was less in patients who had TEA (78.6, R: 0-228.5) vs. IVPCA (105.2, R: 33.9-267.3), $\mathrm{p}=0.0322$. Additionally, fewer patients in the TEA (42.5\%) group experienced inadequate pain control compared to those in the IV-PCA (61.8\%) group, $\mathrm{p}=0.0747$. Figure 2 graphically depicts the pain experience in the TEA group when compared to IV-PCA. After adjustment of preoperative clinical features and perioperative factors, multivariate regression identified older age (Coefficient: 1.18, Standard Error 0.42, $\mathrm{p}=0.006$ ) and use of TEA (coefficient: 25.8 , Standard Error: 10.38, $\mathrm{p}=0.0142$ ) to be independently associated with a lower AUC 48.

\section{Postoperative Surgical and Analgesic Related Complications}

Among 140 patients, 50 (35.7\%) patients experienced a surgical complication, with 10 (7.1\%) of these patients experiencing a severe complication. Collectively, there were 1 neurologic, 8 cardiac, 3 respiratory, 5 renal, 14 gastrointestinal, 8 wound, and 7 other complications. For liver related complications, there were 5 (3.6\%) patients with bile leak and $1(0.7 \%)$ patient who had liver failure. There were zero symptomatic deep vein thrombosis (DVT) events, but two patients were diagnosed with pulmonary embolism in the absence of DVT. Comparing TEA and IV-PCA groups, severe surgical complication rates were similar ( $37.7 \%$ vs. $31.3 \%$, respectively, $\mathrm{p}=0.705$ ). The median peak postoperative bilirubin was 1.4 (R: $0.3 \mathrm{mg} / \mathrm{dL}$ to $11.8 \mathrm{mg} / \mathrm{dL}$ ), and the median peak postoperative creatinine was 0.9 (R: $0.1 \mathrm{mg} / \mathrm{dL}-2.3 \mathrm{mg} / \mathrm{dL}$ ), with no differences in these values between the study groups. Overall, 2 (1.4\%) patients returned to the operating room, and $3(2.1 \%)$ required readmission. The median length of stay was the same between TEA ( 6 days, IQR: 5-7 days) and IV-PCA (6 days, IQR: 5-7 days), $\mathrm{p}=0.957$. There were no 90-day mortalities.

Twelve patients experienced an analgesic related complication that required discontinuation of the original pain control modality. For analgesic related complications, there was no difference between TEA and IV-PCA (12.2\% vs. $2.9 \%$, respectively, $\mathrm{p}=0.187)$, and all were 
less than MAG 3. Two patients with TEA required replacement of the epidural catheter due to catheter migration or malfunction and two TEA group patients required change of pain management modality to IV-PCA due to inadequate pain control. In 15 patients, TEA had to be placed on hold for a median time of 2 hours (IQR: 1-3.25 hours) due to hypotension. Among these patients who experienced hypotension with TEA in place, the median peak creatinine was $0.67 \mathrm{mg} / \mathrm{dL}$ (IQR: $0.6 \mathrm{mg} / \mathrm{dL}-0.84 \mathrm{mg} / \mathrm{dL}$ ) with only one patient developing a peak creatinine above $1.5 \mathrm{mg} / \mathrm{dL}$ during hospitalization.

\section{Postoperative Narcotic Utilization}

The median cumulative OME over the first 5 postoperative days was markedly less in the TEA (155.3 mg, R: 53.43-2747.3 mg) group when compared to IV-PCA (429.8 mg, R: 69.8-1787.5 mg), $\mathrm{p}<0.001$. Additionally, patients in the TEA group required less daily OME for the majority of postoperative days (POD $0-3$, all $\mathrm{p}<0.001$; Figure 3 ). The use of traditional adjuvant agents, such as acetaminophen and ketorolac, was equally distributed between groups.

\section{Patient-reported Outcomes}

The vast majority of patients completed the SF-8 before (TEA: 99.1\%; IV-PCA 100\%) and after (TEA: 90.1\%; IV-PCA 94.1\%) surgery. Using this tool, TEA and IV-PCA patients were found to have similar levels of baseline (preoperative) pain (Table 2), $\mathrm{p}=0.468$; in contrast, postoperatively, a significantly larger number of patients in the TEA (62.5\%) group reported having less than mild pain when compared to the IV-PCA (40.6\%) group, $\mathrm{p}=0.03$.

The SF-36 was answered by $85.8 \%$ of TEA and $79.4 \%$ of IV-PCA patients. While average calculated preoperative freedom from pain scores from the SF-36 were similar between TEA $(90.69 \pm 15.96)$ vs. IV-PCA $(85.38 \pm 19.62) \mathrm{p}=0.159$, postoperative freedom from pain scores were higher in patients with TEA $(37.11 \pm 24.64)$ than patients with IV-PCA (25.93 \pm 16.74 ), $\mathrm{p}=0.010$. After adjustment of preoperative clinical features and perioperative factors, multivariate regression identified TEA (Coefficient: -12.92, Standard Error 5.41, $\mathrm{p}=0.019)$ to be the sole factor associated with less negative change in pain score between preoperative evaluation and discharge.

Likewise, SF-36 negative change in average fatigue/energy scores was less in the TEA $(21.91 \pm 15.70)$ group when compared to the IV-PCA $(32.93 \pm 17.01)$ group, $\mathrm{p}=0.002$. Multivariate linear regression analysis for factors independently associated with less negative change in fatigue identified patients with TEA (Coefficient: -8.98 , Standard Error $3.14, \mathrm{p}=0.005$ ) and patients with a resection less than a major hepatectomy (Coefficient: -9.09, Standard Error 2.71, $\mathrm{p}=0.001$ ).

The HORS instrument was answered by the majority of patients on each post-operative day (POD 1: $91.4 \%$, POD 2: 88.6\%, POD 3 86.4\%, POD 4: 82.4\%, POD $583.3 \%$ ). This instrument identified greater patient satisfaction with pain control in the TEA group compared to the IV-PCA group ( $\mathrm{p}=0.022$, Figure 4 ). Further analysis with a mixed effects model to control for covariates identified a strong association between TEA and patient satisfaction with pain control, $\mathrm{p}=0.035$. 


\section{DISCUSSION}

As short-term hepatobiliary surgical outcomes have improved, focus is shifting to patient experience and oncologic outcomes. Regarding the important aspect of optimization of perioperative analgesia, the hepatobiliary surgical literature includes a number of nonrandomized studies suggesting that TEA is a more complex analgesic regimen that, when inadequately supported at the bedside, can be associated with safety concerns including hypotension and a need for excessive fluid and blood product administration.[22-28] The efficacy of the pain control associated with this modality has been observed to be as good or better than intravenous narcotics. One small randomized trial, comparing TEA to IV-PCA, demonstrated superior pain control in the epidural arm but with a longer time to fulfill discharge criteria. Furthermore, analgesia in the PCA arm was augmented with continuous infiltration of local anesthetic using wound catheters and patient recovery beyond length of stay was not measured.[45] Given the lack of randomized data on this important topic, it is clear that further investigation was warranted.

In the current study a patient cohort, treated mainly with open liver resection, was randomized to TEA vs. IV-PCA with a primary endpoint of pain score AUC over the first 48 hours after surgery, and secondary endpoints of narcotic usage and patient-reported satisfaction, pain control and function. TEA-treated patients experienced similar rates of complications, length of stay and mortality with no statistically or clinically significant increase in hypotensive episodes, transfusion, or renal insufficiency events. Moreover, the pain control experience associated with TEA was superior, particularly during the first 48 hours after surgery. Although not specifically addressing pain level at rest in comparison to during activity, this methodology did include simultaneous evaluation of pain level and function. Ultimately, the primary endpoint was confirmed on four scales, including AUC of pain scores, severe pain event rates, total narcotic usage and patient-reported outcomes of satisfaction with pain control and function.

Several areas of the study require specific commentary. First, the randomization strategy was 2.5 TEA to 1 IV-PCA entrant. The rationale for this methodology was to simultaneously have the statistical power to comment on the primary endpoint of AUC 48, as well as, to comment on potential TEA-related anesthetic complications, including hypotension, transfusion and renal insufficiency. By weighting the accrual toward the TEA group the study was able to determine that the TEA was both effective and safe.

Second, with specific regard to the TEA regimen, the study utilized a strict protocol for drug concentration, infusion, and boluses. The concentration of local anesthetic was $0.075 \%$ bupivacaine, a concentration that is lower than typically reported. Using this lower concentration and a regimented rescue bolus regimen, study patients achieved excellent pain control, while avoiding hypotension and other commonly reported anesthetic complications that have been associated with TEA catheters. The data, therefore, support a reevaluation of prior studies that found excessive rates of hypotension and/or fluid resuscitation in the absence of reporting epidural concentration data, variability in concentration and drug choice, or higher concentrations of local anesthetic.[28] 
Third, within the literature on this topic this study uniquely included patient-reported outcomes data in addition to patient-reported pain scores. These data are important because they confirm that the patient satisfaction and experience associated with improved pain scores was superior in the TEA group. Furthermore, the patient function in the TEA group was superior to the IV-PCA group. This finding is related not only to lower pain levels and less concomitant anxiety, but also to the almost 3-fold reduction in narcotic usage in the TEA group.

This reduction in IV and enteral narcotics is important, as the United States grapples with a significant narcotic dependence that is frequently initiated during a procedure or surgery.[46] Furthermore, in oncologic care there is emerging data that many cancers have high levels of mu opioid receptor expression, $[47,48]$ that when activated by opiate narcotics could potentially mediate tumor growth and spread.[49,50] As more is learned about the interaction between these receptors and perioperative narcotics, it may become imperative that oncologic surgeons seek alternative methods to reduce perioperative opioid delivery. This trial supports that TEA is a safe and effective tool for limiting opiate narcotic dosing.

Fourth, like any randomized trial, global progress in the field does not wait for published results. During the course of this study, enhanced recovery and transversus abdominus plane (TAP) block have emerged as advances in multiple areas of surgical care. Although this study was pathway driven with a modern protocol containing several progressive elements common to enhanced recovery (eg. minimization of tubes and drains), specific oral nonopioid analgesic agents common to many enhanced recovery pathways were not given. Regarding, more traditional adjuvant agents, such as acetaminophen and ketorolac, the distribution of use was equivalent in each group.

Lastly, it is clear that minimally invasive approaches provide pain and experience advantages to patients. However, a recent national audit of 2015-2016 activity found that the vast majority of liver resections (84\%) continue to be done through open approaches, with a higher penetrance of minimally invasive approaches in minor liver resection (78\%), compared to major liver resection (95\%) (unpublished ACS-NSQIP HPB collaborative data courtesy of Dr. Henry Pitt). Although each of these recent trends was not included in this trial, the results validate the shared concepts of the trial, which focused on the still most common (open) surgical approach and established that regional block is superior to IV-PCA based analgesic strategies. The TEA-based strategy now forms a standard-of-care for subsequent randomized trial comparison to other regional blocks, such as TAP, with enhanced recovery adjuvants in both arms. Preferably such a trial would be performed in a multi-center fashion, however the use of multiple metrics of patient experience with pain control, including patient reported outcomes, facilitates the detection of substantial differences between modalities, obviating the need for large (ie. thousands of patients) cohorts.

In summary, this randomized trial, which focused mainly on liver surgery patients, finds that TEA provides a superior pain control experience, with no deficit in surgical complications, anesthetic complications, length of stay, or mortality. 


\section{Acknowledgments}

The authors would like to recognize the significant contributions of Ms. Ruth Haynes (administrative support), Ms. Theresa Honey, BS (Senior Research Data Coordinator), Ms. Rose Joan D Erfe, BS CCRC (Research Coordinator Clinical Studies), Wei Qiao MS PhD (analyst in Department of Biostatistics.), and the MD Anderson Department of Anesthesiology and Perioperative Medicine Acute Pain Service.

Funding Sources: Dr. Bradford Kim is supported by National Institutes of Health grant T32CA009599; Patient Reported Outcomes analysis was supported by NIH/NCI, R01CA205146 to Dr. Xin Shelley Wang

\section{References}

1. Aloia TA, Fahy BN, Fischer CP, et al. Predicting poor outcome following hepatectomy: analysis of 2313 hepatectomies in the NSQIP database. HPB (Oxford). 2009; 11:510-515. [PubMed: 19816616]

2. Tzeng CW, Cooper AB, Vauthey JN, et al. Predictors of morbidity and mortality after hepatectomy in elderly patients: analysis of 7621 NSQIP patients. HPB (Oxford). 2014; 16:459-468. [PubMed: 24033514]

3. Adams RB, Aloia TA, Loyer E, et al. Selection for hepatic resection of colorectal liver metastases: expert consensus statement. HPB (Oxford). 2013; 15:91-103. [PubMed: 23297719]

4. Madoff DC, Abdalla EK, Gupta S, et al. Transhepatic ipsilateral right portal vein embolization extended to segment IV: improving hypertrophy and resection outcomes with spherical particles and coils. J Vasc Interv Radiol. 2005; 16:215-225. [PubMed: 15713922]

5. Kishi Y, Zorzi D, Contreras CM, et al. Extended Preoperative Chemotherapy Does Not Improve Pathologic Response and Increases Postoperative Liver Insufficiency After Hepatic Resection for Colorectal Liver Metastases. Annals of Surgical Oncology. 2010; 17:2870-2876. [PubMed: 20567921]

6. Aloia TA, Zorzi D, Abdalla EK, Vauthey J-N. Two-Surgeon Technique for Hepatic Parenchymal Transection of the Noncirrhotic Liver Using Saline-Linked Cautery and Ultrasonic Dissection. Annals of Surgery. 2005; 242:172-177. [PubMed: 16041206]

7. Gold JS, Are C, Kornprat P, et al. Increased Use of Parenchymal-Sparing Surgery for Bilateral Liver Metastases From Colorectal Cancer Is Associated With Improved Mortality Without Change in Oncologic Outcome: Trends in Treatment Over Time in 440 Patients. Annals of Surgery. 2008; 247:109-117. [PubMed: 18156930]

8. Karanjia ND, Lordan JT, Quiney N, et al. A comparison of right and extended right hepatectomy with all other hepatic resections for colorectal liver metastases: a ten-year study. Eur J Surg Oncol. 2009; 35:65-70. [PubMed: 18222623]

9. Redaelli CA, Wagner M, Krahenbuhl L, et al. Liver surgery in the era of tissue-preserving resections: early and late outcome in patients with primary and secondary hepatic tumors. World J Surg. 2002; 26:1126-1132. [PubMed: 12209242]

10. Sarpel U, Bonavia AS, Grucela A, et al. Does Anatomic Versus Nonanatomic Resection Affect Recurrence and Survival in Patients Undergoing Surgery for Colorectal Liver Metastasis? Annals of Surgical Oncology. 2009; 16:379-384. [PubMed: 19020941]

11. Cameron JL, Pitt HA, Yeo CJ, et al. One hundred and forty-five consecutive pancreaticoduodenectomies without mortality. Ann Surg. 1993; 217:430-435. discussion 435-438. [PubMed: 8098202]

12. Fernandez-del Castillo C, Rattner DW, Warshaw AL. Standards for pancreatic resection in the 1990s. Arch Surg. 1995; 130:295-299. discussion 299-300. [PubMed: 7887797]

13. Lieberman MD, Kilburn H, Lindsey M, Brennan MF. Relation of perioperative deaths to hospital volume among patients undergoing pancreatic resection for malignancy. Ann Surg. 1995; 222:638-645. [PubMed: 7487211]

14. Gordon TA, Burleyson GP, Tielsch JM, Cameron JL. The effects of regionalization on cost and outcome for one general high-risk surgical procedure. Ann Surg. 1995; 221:43-49. [PubMed: 7826160] 
15. Merkow J, Paniccia A, Edil BH. Laparoscopic pancreaticoduodenectomy: a descriptive and comparative review. Chin J Cancer Res. 2015; 27:368-375. [PubMed: 26361406]

16. Aly MY, Tsutsumi K, Nakamura M, et al. Comparative study of laparoscopic and open distal pancreatectomy. J Laparoendosc Adv Surg Tech A. 2010; 20:435-440. [PubMed: 20518689]

17. Nguyen KT, Marsh JW, Tsung A, et al. Comparative benefits of laparoscopic vs open hepatic resection: a critical appraisal. Arch Surg. 2011; 146:348-356. [PubMed: 21079109]

18. Apfelbaum JL, Chen C, Mehta SS, Gan TJ. Postoperative pain experience: results from a national survey suggest postoperative pain continues to be undermanaged. Anesth Analg. 2003; 97:534540. table of contents. [PubMed: 12873949]

19. Holte K, Kehlet H. Effect of postoperative epidural analgesia on surgical outcome. Minerva Anestesiol. 2002; 68:157-161. [PubMed: 12024074]

20. Kehlet H, Holte K. Effect of postoperative analgesia on surgical outcome. Br J Anaesth. 2001; 87:62-72. [PubMed: 11460814]

21. Aloia TA, Zimmitti G, Conrad C, et al. Return to intended oncologic treatment (RIOT): a novel metric for evaluating the quality of oncosurgical therapy for malignancy. J Surg Oncol. 2014; 110:107-114. [PubMed: 24846705]

22. Allen S, DeRoche A, Adams L, et al. Effect of epidural compared to patient-controlled intravenous analgesia on outcomes for patients undergoing liver resection for neoplastic disease. J Surg Oncol. 2017

23. Ahn JH, Ahn HJ. Effect of thoracic epidural analgesia on recovery of bowel function after major upper abdominal surgery. J Clin Anesth. 2016; 34:247-252. [PubMed: 27687384]

24. Oh TK, Lim MC, Lee Y, et al. Improved Postoperative Pain Control for Cytoreductive Surgery in Women With Ovarian Cancer Using Patient-Controlled Epidural Analgesia. Int J Gynecol Cancer. 2016; 26:588-593. [PubMed: 26825838]

25. Shi WZ, Miao YL, Yakoob MY, et al. Recovery of gastrointestinal function with thoracic epidural vs. systemic analgesia following gastrointestinal surgery. Acta Anaesthesiol Scand. 2014; 58:923932. [PubMed: 25060245]

26. Toren P, Ladak S, Ma C, et al. Comparison of epidural and intravenous patient controlled analgesia in patients undergoing radical cystectomy. Can J Urol. 2009; 16:4716-4720. [PubMed: 19671221]

27. Bartha E, Carlsson P, Kalman S. Evaluation of costs and effects of epidural analgesia and patientcontrolled intravenous analgesia after major abdominal surgery. Br J Anaesth. 2006; 96:111-117. [PubMed: 16257994]

28. Page A, Rostad B, Staley CA, et al. Epidural analgesia in hepatic resection. J Am Coll Surg. 2008; 206:1184-1192. [PubMed: 18501817]

29. Pang YY. The Brisbane 2000 terminology of liver anatomy and resections. HPB. 2000; 2:333-39. HPB (Oxford) 2002;4:99; author reply 99-100.

30. Couinaud C. Liver lobes and segments: notes on the anatomical architecture and surgery of the liver. Presse Med. 1954; 62:709-712. [PubMed: 13177441]

31. Couinaud C. Surgical anatomy of the liver Several new aspect. Chirurgie. 1986; 112:337-342. [PubMed: 3608701]

32. Couinaud C. The anatomy of the liver. Ann Ital Chir. 1992; 63:693-697. [PubMed: 1305370]

33. Nielsen S, Degenhardt L, Hoban B, Gisev N. A synthesis of oral morphine equivalents (OME) for opioid utilisation studies. Pharmacoepidemiol Drug Saf. 2016; 25:733-737. [PubMed: 26693665]

34. Porembka MR, Hall BL, Hirbe M, Strasberg SM. Quantitative weighting of postoperative complications based on the accordion severity grading system: demonstration of potential impact using the american college of surgeons national surgical quality improvement program. J Am Coll Surg. 2010; 210:286-298. [PubMed: 20193891]

35. Brooke-Smith M, Figueras J, Ullah S, et al. Prospective evaluation of the International Study Group for Liver Surgery definition of bile leak after a liver resection and the role of routine operative drainage: an international multicentre study. HPB (Oxford). 2015; 17:46-51. [PubMed: 25059275]

36. Rahbari NN, Garden OJ, Padbury R, et al. Posthepatectomy liver failure: a definition and grading by the International Study Group of Liver Surgery (ISGLS). Surgery. 2011; 149:713-724. [PubMed: 21236455] 
37. Roberts B, Browne J, Ocaka KF, et al. The reliability and validity of the SF-8 with a conflictaffected population in northern Uganda. Health Qual Life Outcomes. 2008; 6:108. [PubMed: 19055716]

38. Bost JE, Williams BA, Bottegal MT, et al. The 8-item Short-Form Health Survey and the physical comfort composite score of the quality of recovery 40 -item scale provide the most responsive assessments of pain, physical function, and mental function during the first 4 days after ambulatory knee surgery with regional anesthesia. Anesth Analg. 2007; 105:1693-1700. table of contents. [PubMed: 18042869]

39. Sugimoto M, Takegami M, Suzukamo Y, et al. Health-related quality of life in Japanese men with localized prostate cancer: assessment with the SF-8. Int J Urol. 2008; 15:524-528. [PubMed: 18422571]

40. Ware JE Jr, Sherbourne CD. The MOS 36-item short-form health survey (SF-36). I. Conceptual framework and item selection. Med Care. 1992; 30:473-483. [PubMed: 1593914]

41. McHorney CA, Ware JE Jr, Lu JF, Sherbourne CD. The MOS 36-item Short-Form Health Survey (SF-36): III: Tests of data quality, scaling assumptions, and reliability across diverse patient groups. Med Care. 1994; 32:40-66. [PubMed: 8277801]

42. McHorney CA, Ware JE Jr, Raczek AE. The MOS 36-Item Short-Form Health Survey (SF-36): II: Psychometric and clinical tests of validity in measuring physical and mental health constructs. Med Care. 1993; 31:247-263. [PubMed: 8450681]

43. Gan TJ, Joshi GP, Viscusi E, et al. Preoperative parenteral parecoxib and follow-up oral valdecoxib reduce length of stay and improve quality of patient recovery after laparoscopic cholecystectomy surgery. Anesth Analg. 2004; 98:1665-1673. table of contents. [PubMed: 15155324]

44. Shamseer L, Hopewell S, Altman DG, et al. Update on the endorsement of CONSORT by high impact factor journals: a survey of journal "Instructions to Authors" in 2014. Trials. 2016; 17:301. [PubMed: 27343072]

45. Revie EJ, McKeown DW, Wilson JA, et al. Randomized clinical trial of local infiltration plus patient-controlled opiate analgesia vs. epidural analgesia following liver resection surgery. HPB (Oxford). 2012; 14:611-618. [PubMed: 22882198]

46. Maxwell JC. The prescription drug epidemic in the United States: a perfect storm. Drug Alcohol Rev. 2011; 30:264-270. [PubMed: 21545556]

47. Bortsov AV, Millikan RC, Belfer I, et al. mu-Opioid receptor gene A118G polymorphism predicts survival in patients with breast cancer. Anesthesiology. 2012; 116:896-902. [PubMed: 22433205]

48. Wang S, Li Y, Liu XD, et al. Polymorphism of A118G in mu-opioid receptor gene is associated with risk of esophageal squamous cell carcinoma in a Chinese population. Int J Clin Oncol. 2013; 18:666-669. [PubMed: 22752309]

49. Lennon FE, Mirzapoiazova T, Mambetsariev B, et al. The Mu opioid receptor promotes opioid and growth factor-induced proliferation, migration and Epithelial Mesenchymal Transition (EMT) in human lung cancer. PLoS One. 2014; 9:e91577. [PubMed: 24662916]

50. Lennon FE, Mirzapoiazova T, Mambetsariev B, et al. Overexpression of the mu-opioid receptor in human non-small cell lung cancer promotes Akt and mTOR activation, tumor growth, and metastasis. Anesthesiology. 2012; 116:857-867. [PubMed: 22343475] 


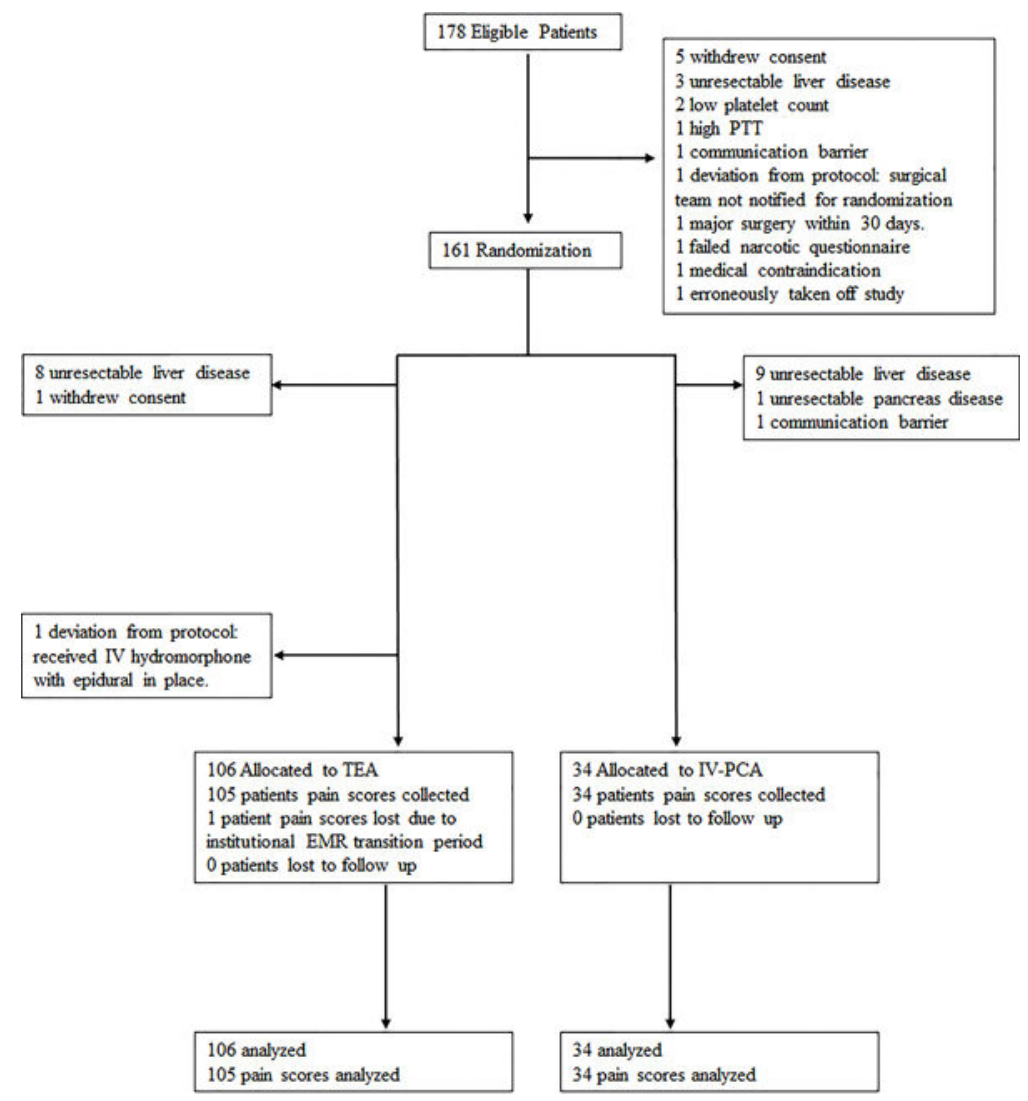

Figure 1.

Consort Flow Diagram 


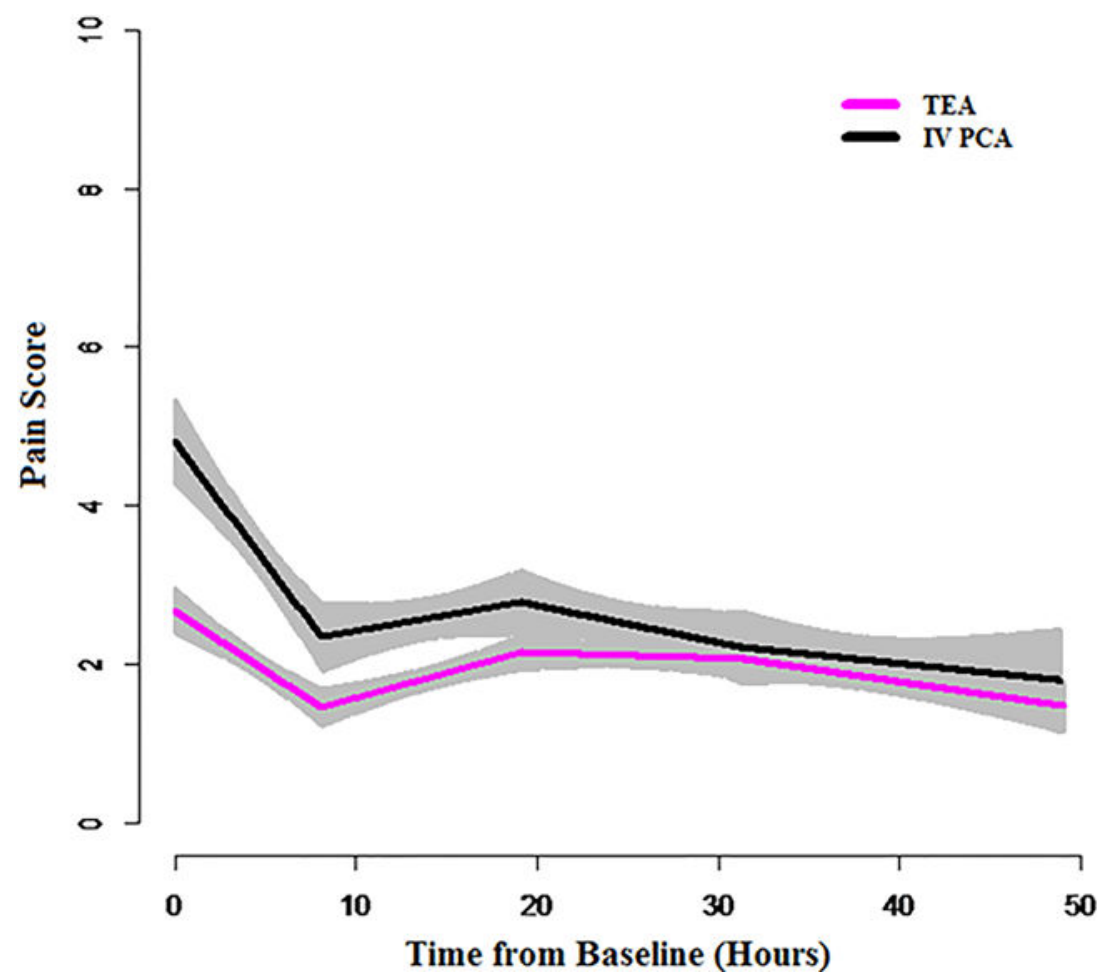

Figure 2.

Pain Scores Over Time in Thoracic Epidural Analgesia (TEA) vs. Intravenous Patient Controlled Analgesia (IV-PCA). The depicted 95\% confidence bands represent interval estimators that characterize the extent of uncertainty for mean estimation of pain score at significance level 0.05 over the 48-hour follow-up time. 

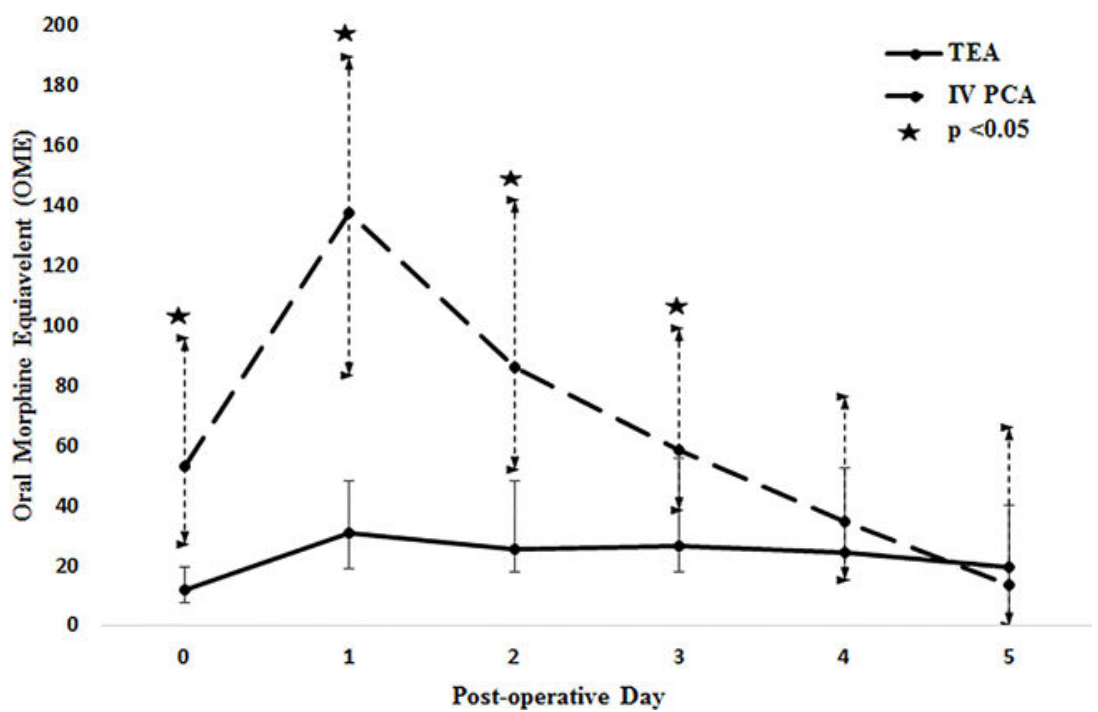

Figure 3.

Median Oral Morphine Equivalent (with Interquartile Range) Used on Each Postoperative Day in Thoracic Epidural Analgesia (TEA) vs. Intravenous Patient Controlled Analgesia (IV-PCA) 


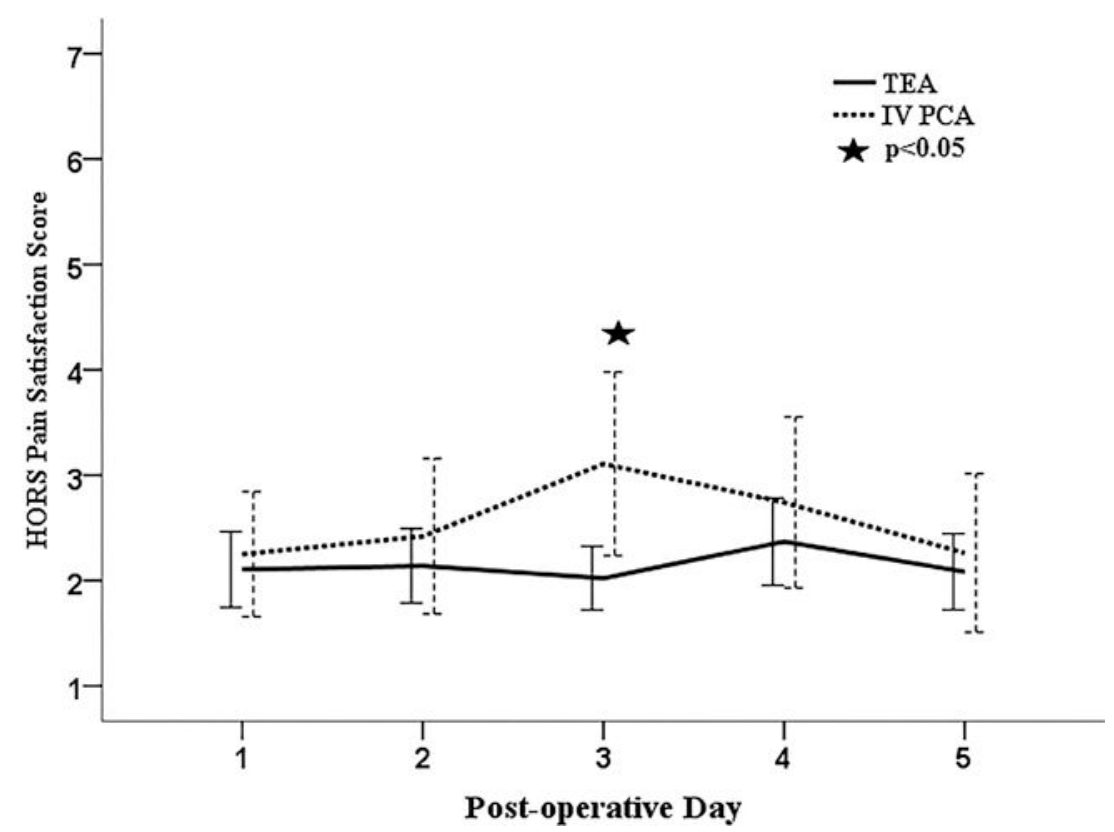

Figure 4.

Patient Satisfaction with Thoracic Epidural Analgesia (TEA) vs. Intravenous Patient Controlled Analgesia (IV-PCA) measured by the Health Outcomes Recovery Survey (HORS): Lower score indicates better patient reported pain satisfaction. 


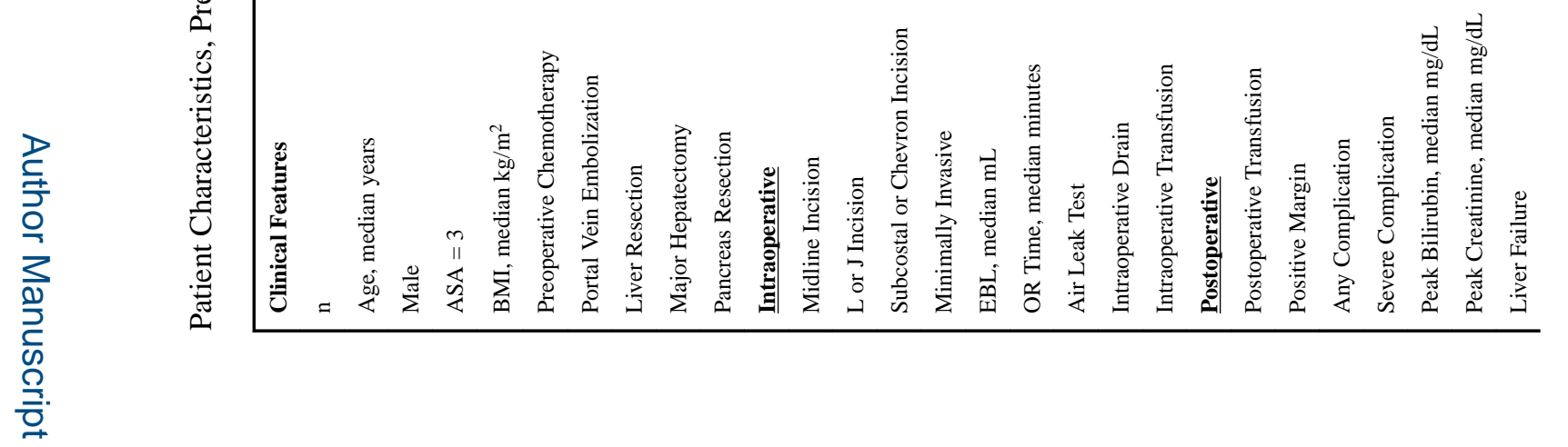
Ann Surg. Author manuscript; available in PMC 2018 September 01. 


\section{Table 2}

Patient-reported pain from SF-8 before and after surgery.

\begin{tabular}{|c|c|c|c|c|}
\hline & TEA & $\%$ & IV-PCA & $\%$ \\
\hline \multicolumn{5}{|c|}{ Preoperative Pain } \\
\hline None & 64 & 61.0 & 16 & 47.1 \\
\hline Very Mild & 25 & 23.8 & 9 & 26.5 \\
\hline Mild & 8 & 7.6 & 6 & 17.7 \\
\hline Moderate & 5 & 4.8 & 2 & 5.9 \\
\hline Severe & 3 & 2.9 & 1 & 2.9 \\
\hline Very Severe & 0 & 0.0 & 0 & 0.0 \\
\hline \multicolumn{5}{|l|}{ POD 1 Pain } \\
\hline None & 33 & 34.4 & 10 & 31.3 \\
\hline Very Mild & 27 & 28.1 & 3 & 9.4 \\
\hline Mild & 13 & 13.5 & 5 & 15.6 \\
\hline Moderate & 15 & 15.6 & 6 & 18.8 \\
\hline Severe & 6 & 6.3 & 5 & 15.6 \\
\hline Very Severe & 2 & 2.1 & 3 & 9.4 \\
\hline
\end{tabular}

Abbreviations: IV-PCA, Intravenous Patient Controlled Analgesia; TEA, Thoracic Epidural Analgesia; POD, Post-operative 


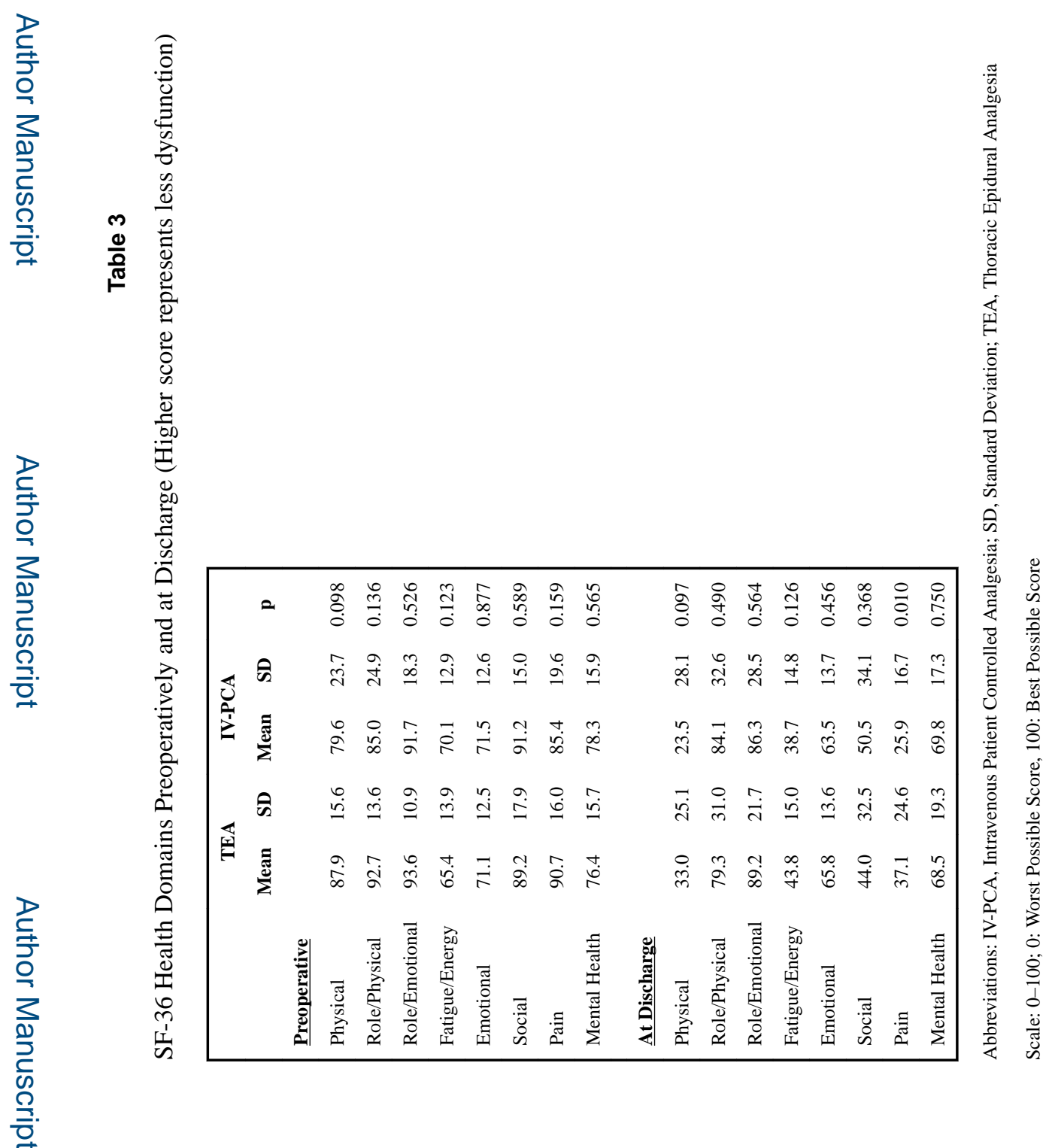

Ann Surg. Author manuscript; available in PMC 2018 September 01. 